\title{
Dialogando sobre pertenencia étnica con docentes bribris y cabécares de Talamanca: experiencias del trabajo colaborativo
}

\section{Federico Guevara Víquez ${ }^{1}$ Sandra Ovares Barquero²}

Recepción: 26 de marzo de 2014 / Aprobación: 17 de junio de 2014

\section{Resumen}

Este artículo constituye uno de los productos del proyecto de extensión "Reconstrucción de Saberes" de la División de Educación Rural (DER) de la Universidad Nacional (UNA) que involucra el trabajo participativo y colaborativo con maestros indígenas de Lengua y Cultura de la región indígena de Talamanca, en la vertiente atlántico sur de Costa Rica. En concreto, se expone la dinámica participativa desarrollada entre investigadores de la DER en conjunto con los maestros para el abordaje y análisis de la identidad cultural y la cultura entre bribris y cabécares. Al final, se plantean algunas reflexiones en torno a las posibilidades reales de diálogo entre el conocimiento vernáculo de las comunidades y el saber científico generado en las universidades.

\section{Palabras clave}

Cultura; identidad étnica; bribris; cabécares; docentes indígenas

\section{Abstract}

This article is one of the products of the project "Reconstruction of Knowledge" of the Division of Rural Education (DER) from the National University of Costa Rica (UNA). This project involves participatory and collaborative work with indigenous teachers of Language and Culture from Talamanca, indigenous region of Costa Rica's southern Atlantic side. Specifically, participatory dynamics developed between DER researchers and teachers to approach and analyze cultural identity and culture among Cabecar and Bribri natives are presently exposed. Finally, some reflections on the real dialog possibilities between the communities' vernacular knowledge and the scientific knowledge generated in universities are provided.

1 Costarricense. Máster en Antropología por la Universidad de Costa Rica (UCR). Profesor e Investigador de la División de Educación Rural (DER) del Centro de Investigación y Docencia en Educación (CIDE) de la Universidad Nacional de Costa Rica (UNA). Correo electrónico: feguevi@gmail.com

2 Costarricense. Máster en Formación de Formadores por la UNA. Vicedecana del CIDE de la UNA. Correo electrónico: sandra.ovares.barquero@una.cr 


\section{Keywords}

Culture, ethnicity; Bribri; Cabecar; indigenous teachers

\section{Resumo}

Este artigo é um dos produtos do projeto de extensão "Reconstrução do Conhecimento", da Divisão de Educação Rural (DER) da Universidade Nacional (UNA), envolvendo o trabalho participativo e colaborativo com professores indígenas de língua e cultura da região indiana Talamanca, na vertente atlântica do sul da Costa Rica. Especificamente, a dinâmica participativa desenvolvida entre pesquisadores e professores para DER abordagem e análise da identidade cultural e da cultura entre Cabecares Bribri e exposto. Finalmente algumas reflexões sobre as reais possibilidades de diálogo entre o conhecimento vernacular comunidade eo conhecimento científico gerado nas universidades são dadas.

\section{Palavras chave}

Cultura; identidade; Bribri; Cabecar; professores indígenas

\section{Introducción}

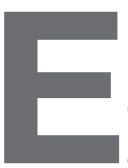

I presente trabajo constituye uno de los productos del proyecto de extensión número 0472-10 "Reconstrucción de Saberes" adscrito a la División de Educación Rural (DER), del Centro de Investigación y Docencia en Educación (CIDE) de la Universidad Nacional (UNA), de Costa Rica. Este proyecto involucra el trabajo participativo y colaborativo con maestros indígenas de Lengua y Cultura de la región de Talamanca Bribri y Cabécar, en la vertiente atlántico sur del país. El proyecto en mención busca potenciar capacidades en este tipo de profesionales de la educación, colaborando con la producción de material didáctico y de estrategias educativas para un mejor desenvolvimiento profesional. Además, de manera indirecta, tiene el propósito de incidir positivamente en la mejora de la oferta educativa para la población en edad escolar.

El documento aborda el tema de la identidad cultural, empoderando al docente desde acciones de educación continua con un bagaje conceptual que le permita mejorar su desempeño como educador y como intelectual profesional dentro de su cultura y su comunidad. En esta experiencia específicamente se profundiza en cómo se establece la pertenencia étnica entre los pueblos bribris y cabécares, con el fin de tener una mejor comprensión de este fenómeno y visualizar a futuro mecanismos que coadyuven al fortalecimiento del sentido de identidad no solo de los docentes de Lengua y Cultura, sino de la niñez en edad escolar. Asimismo el empoderamiento por parte de 
los profesionales en educación de categorías analíticas para la mejor comprensión de fenómenos, como la cultura y la identidad, son pasos necesarios para mejorar procesos de Educación Intercultural Bilingüe (EIB) que faculten una relación horizontal y complementaria entre distintas culturas que cohabitan en un mismo país, donde el aprendiente adquiera competencias que le permitan desempeñarse en dos o más culturas, sin menoscabo del acervo cultural propio y lingüístico que lo caracteriza.

Parte del documento se construye a partir de las experiencias vividas con los maestros de Lengua y Cultura bribris y cabécares de la región de Talamanca. A partir de un proceso dialógico se complementaron, por un lado, la visión propia de estos maestros acerca de lo que entienden por cultura e identidad, y por el otro, el de los investigadores del proyecto con su bagaje teórico y científico sobre estos fenómenos, para la generación de una mirada reflexiva conjunta. Finalmente, se aportan algunas otras reflexiones sobre el papel de los investigadores en propiciar espacios de diálogo entre el conocimiento tradicional, el punto de vista de las comunidades indígenas y el conocimiento generado por los centros de educación superior relacionado con estas mismas poblaciones, en aras de generar procesos de construcción colectiva del conocimiento.

\section{Los pueblos indígenas bribris y cabécares, y los maestros de Lengua y Cultura}

Los pueblos bribri y cabécar habitan mayoritariamente en distintas regiones de la Cordillera de Talamanca. Según el último Censo Nacional realizado en Costa Rica en el año 2011 (INEC, 2014), la población indígena es de 104 143 individuos, por lo cual representan poco más del 2\% de la población nacional. Existen ocho pueblos indígenas diferentes, distribuidos en 24 territorios determinados por ley. La población indígena bribri total del país según el mismo censo asciende a los 18198 individuos, mientras que los cabécares suman 16985 individuos.

Desde el punto de vista lingüístico, los idiomas bribri y cabécar son de estirpe chibchense (Constenla, 1991). La diferencia entre ambas lenguas es semejante a la diferencia que puede existir entre el portugués y el español. En términos generales, los bribris mantienen la lengua materna en un 45\% mientras que los cabécares, un $74 \%$, porcentajes que oscilan hacia arriba o hacia abajo según el territorio indígena de que se trate. Los bribris viven, de manera primordial, en los territorios indígenas de Talamanca Bribri y Keköldi en el caribe sur del país, y en los territorios de Cabagra y Salitre en el pacífico sur. Por su parte, la población cabécar vive mayoritariamente en los territorios de Talamanca Cabécar, Alto Chirripó, Bajo Chirripó, Tayni, Telire, Nairi awari en la vertiente atlántica del país, y en los territorios de Ujarrás y China Kichá, en el pacífico sur del país. 
En el marco de este proyecto, hemos abordado el trabajo conjunto con maestros de Lengua y Cultura que se desempeñan en los territorios indígenas de Talamanca Bribri, Talamanca Cabécar (en el valle de Talamanca) y Keköldi. Según el Censo Nacional del año 2011, en el caso de Talamanca Bribri la lengua indígena se mantiene en un 60,8\% de la población, en el caso de Talamanca Cabécar el procentaje es de 64,9\% y para el caso de Tayní es de 86,7\% mientras que en Keköldi este cae a un 36,3\%.

En una ponencia previa (Guevara y Ovares, 2013), hemos abordado brevemente los antecedentes del sistema actual de EIB aplicado en Costa Rica. El antecedente más inmediato sobre dicho sistema de educación indígena se da en 1996, cuando se plantean y proponen los programas de Lengua y Cultura para el I y II ciclo de la educación primaria, los cuales aún se siguen desarrollando en las escuelas indígenas del país. Otro elemento importante de resaltar con respecto al sistema de educación para territorios indígenas es que por parte del Ministerio de Educación Pública (MEP), en el año 2009, se creó la primera Dirección Regional Educativa de carácter indígena: la Región Educativa de Sula', que abarca los territorios indígenas bribris y cábecares de la región atlántica de Talamanca.

Los programas de Lengua y Cultura son, hasta el momento, el elemento que particulariza la oferta educativa diferenciada para la población infantil de territorios indígenas dentro del sistema de educación pública costarricense. Dichos programas son ejecutados por los maestros de Lengua y Cultura, figura creada para hacerse cargo de esta faena, bajo la supervisión y asesoramiento del Departamento de Educación Indígena del MEP. Luego de varios decretos ejecutivos, hoy el Departamento de Educación Indígena se ha reestructurado en el Departamento de Educación Intercultural y la Unidad de Educación Indígena es la que se encarga de la supervisión y asesoramiento de los programas de Lengua y Cultura.

Dentro de esta estructura, los docentes de Lengua y Cultura tienen una serie de características que, a su vez, los particularizan, las cuales son necesarias referir para comprender los alcances y limitaciones de la EIB indígena de Costa Rica. En primer lugar, el nombramiento de estos docentes se realiza a través de un código de "docente de apoyo" que la mayoría de las veces tiene carácter itinerante; es decir, un docente de Lengua o Cultura tiene que fungir en más de una escuela. Otra característica es que para el caso de las clases de Lengua, se imparten solo tres lecciones semanales por nivel, mientras que para la materia de Cultura son dos lecciones semanales por nivel. En segundo lugar, estos maestros no poseen preparación profesional, salvo contadas excepciones; para el año 2006, el 90\% de estos docentes solo tenían el $6^{\circ}$ año de primaria aprobado (UNICEF, 2006). Relacionado con el punto anterior, los criterios de selección de este personal están más determinados por el dominio de la lengua indígena así como también de conocimientos o saberes tradicionales propios de cada pueblo indígena. Finalmente, en el nombramiento anual 
de estos docentes ha tenido cuota de participación la Asociación de Desarrollo Integral en cada territorio indígena, en calidad de entidad de mayor autoridad en los territorios indígenas, de acuerdo con la legislación nacional vigente ${ }^{3}$.

Nosotros nos adherimos al modelo ideal de ElB que implica "una educación enraizada en la cultura de referencia inmediata de los educandos pero abierta a la incorporación de elementos y contenidos provenientes de otros horizontes culturales" (López, 2001, p. 7). La ElB promueve una educación en la que se aprovechan dos idiomas (un idioma materno y un segundo idioma) de manera equilibrada como instrumentos y vehículos de aprendizaje. Apreciaciones más de fondo sobre la conceptualización y las características de la EIB así como del sistema de educativo que se ha implementado en Costa Rica serán abordadas en ulteriores publicaciones del proyecto en cuestión. De momento, se puede afirmar que en el ámbito nacional no hemos desarrollado completamente las posibilidades que ofrece el modelo de EIB que se ha dado en otras latitudes de Latinoamérica. En Costa Rica, aún existe la necesidad de contar con maestros profesionales graduados que sean de los propios territorios indígenas, para que trabajen en las escuelas de estas regiones.

Sobre este respecto, ya el MEP ha emitido políticas que dispensan preponderantemente el nombramiento de personas indígenas como maestros en las instituciones educativas de dichas regiones, tal es el caso del decreto ejecutivo № 37801-MEP 2013. Sin embargo, aún queda mucho por mejorar en cuanto a la profesionalización de los docentes de Lengua y Cultura. También existe necesidad de elaborar material didáctico para cada uno de los programas de Lengua y Cultura, para cada una de las lenguas indígenas que aún se practican y para cada uno de los pueblos indígenas según sus características socioculturales propias. Falta más desarrollo curricular de base, de acuerdo con la particularidad de cada pueblo indígena, en aras de desarrollar verdaderos procesos de EIB, que partan de la lengua materna en los lugares donde aún se mantenga, que fomenten el bilingüismo aditivo, que desarrollen los contenidos básicos definidos por la política educativa estatal, sin menoscabo de los saberes y recursos culturales locales. Se han logrado algunas cosas, pero también falta mucho recorrido sobre este respecto. A continuación exponemos los referentes teóricos a partir de los cuales se ha desarrollado el trabajo colaborativo con los maestros de Lengua y Cultura de Talamanca para el abordaje de la cultura, la identidad y la pertenencia étnica.

3 Recientemente, el decreto No 37801-MEP estableció, desde el año 2013, una nueva estructura de participación de las comunidades indígenas en el nombramiento tanto de docentes regulares como de los docentes de Lengua y Cultura para las escuelas, creando la figura de Consejos Consultivos Locales de Educación Indígena, entre otras transformaciones estructurales del sistema de educación formal para pueblos indígenas de Costa Rica. 


\section{Una delimitación conceptual para la identidad étnica y para la cultura: sus diferencias y relaciones}

La identidad cultural ha sido analizada desde múltiples enfoques a lo largo de la historia dependiendo de la disciplina académica. Han existido visiones esencialistas, primordialistas, instrumentalistas, constructivistas e interaccionistas acerca del fenómeno. Asimismo, la identidad cultural puede ser analizada en multiplicidad de contextos, sean estos de relación interétnica horizontal, en contextos de colonialismo, o bien, en lo interno de los Estados-naciones, en contextos de globalización, entre otros. A continuación vamos a delimitar nuestra comprensión sobre este fenómeno colectivo que es protagonizado por distintos grupos culturales a lo largo de la historia de la humanidad.

Como bien lo señala Bartolomé (2006, p. 29), la identidad étnica "se construye a partir de una estructuración ideológica de las representaciones colectivas derivadas de la relación diádica y contrastiva entre un 'nosotros' y un 'los otros'”. Se trata de una construcción que es realizada, ante todo, para expresar una alteridad frente a otras sociedades y para la organización de la conducta. La identidad étnica o cultural implica entonces un proceso de adhesión/exclusión, que discrimina (en su sentido más estricto, como proceso de separación y clasificación) entre semejantes y extraños. De esta forma, a través de los procesos identitarios se establecen y constituyen grupos culturales diferenciados, y a la vez se determinan las posibles formas de interacción entre los distintos grupos establecidos que entran en contacto. Sin embargo, hay que distinguir entre identidad étnica y cultura, las cuales se encuentran muy relacionadas, pero que esencialmente son diferentes:

Es la relación con otras identidades posibles la que genera una necesidad de identificación, culturalmente argumentada, lo que da cuenta del carácter relacional de las identidades colectivas y su dependencia de los variables contextos históricos. Por ello es frecuente que se confunda identidad con cultura, ya que la apelación a la cultura suele desempeñarse como el recurso crucial para afirmar la distintividad, por lo que se confunde el objetivo del discurso (identidad) con los argumentos (cultura) que se utilizan para enunciarlo (Bartolomé, 2006, p. 44).

Bartolomé también hace una aproximación conceptual de la identidad étnica que consideramos idónea:

Por identidad étnica entiendo entonces una construcción ideológica histórica, contingente, relacional, no esencial y eventualmente variable, que manifiesta un carácter procesual y dinámico, y que requiere de referentes culturales para constituirse como tal y enfatizar su singularidad, así como demarcar los límites que la separan de otras identidades posibles (Bartolomé, 2006, p. 44).

A partir de esta comprensión de la identidad étnica, como un proceso de adhesión entre semejantes y diferenciación frente a otredades, es que partimos al análisis de las identidad cultural de bribris y cabécares. Hacemos énfasis en 
que, desde nuestra perspectiva, el elemento crucial de la identidad es su papel para establecer la pertenencia étnica de individuos y grupos humanos.

Los conceptos de identidad y cultura aluden a fenómenos distintos, pero relacionados. Muchas veces, fuera de los círculos académicos que estudian formalmente estos fenómenos sociales, los términos han tendido a ser considerados, erróneamente, como sinónimos y a reducirlos a elementos (materiales o inmateriales) específicos, como lenguaje, rituales y costumbres específicas. Refiriéndonos al caso específico de la educación, estos conceptos son centrales si se quieren desarrollar idóneos procesos de EIB, por lo que los docentes implicados en dichos procesos no deben confundirlos; al contrario, deben tener clara su distinción y relación para evitar caer en prácticas educativas poco provechosas que puedan seguir generando procesos de aculturación (lo que busca evitar la EIB, entre otras cosas) y una "folklorización" del planeamiento curricular que se limita a desarrollar algunas celebraciones de efemérides y representaciones artísticas en determinados momentos del calendario escolar.

Para explicar la diferencia y la relación entre los conceptos de identidad y cultura en el trabajo participativo con los maestros de Lengua y Cultura de Talamanca, hemos partido de la revisión de investigaciones sobre prácticas educativas interculturales en docentes escolares y su comprensión de los conceptos de identidad y cultura, como la investigación de Jiménez (2009) desarrollada en México, la cual constituye un importante referente para nuestro quehacer. Compartimos su caracterización que diferencia la cultura como generadora de procesos de rutinización de prácticas tradicionales, en tanto que la identidad, por su naturaleza dialógica y relacional -en el mismo sentido que le da Bartolomé (2006) - toma algunas de las prácticas rutinizadas por la cultura, para convertirlas en emblemas de contraste. De la exposición teórica de Jiménez derivamos una síntesis gráfica contenida en el Esquema 1, para trabajar los mencionados conceptos con los maestros de Lengua y Cultura en el taller.

¿Quién es bribri?, ¿quién es cabécar?, ¿cuáles son los elementos centrales de la cultura que determinan la pertenencia o no al pueblo bribri y cabécar? Estas son preguntas que problematizan el proceso de diálogo que desarrollamos con los docentes de Lengua y Cultura para llevar reflexionar, de manera conjunta, sobre las bases que fundamentan la pertenencia étnica de estos pueblos indígenas. 


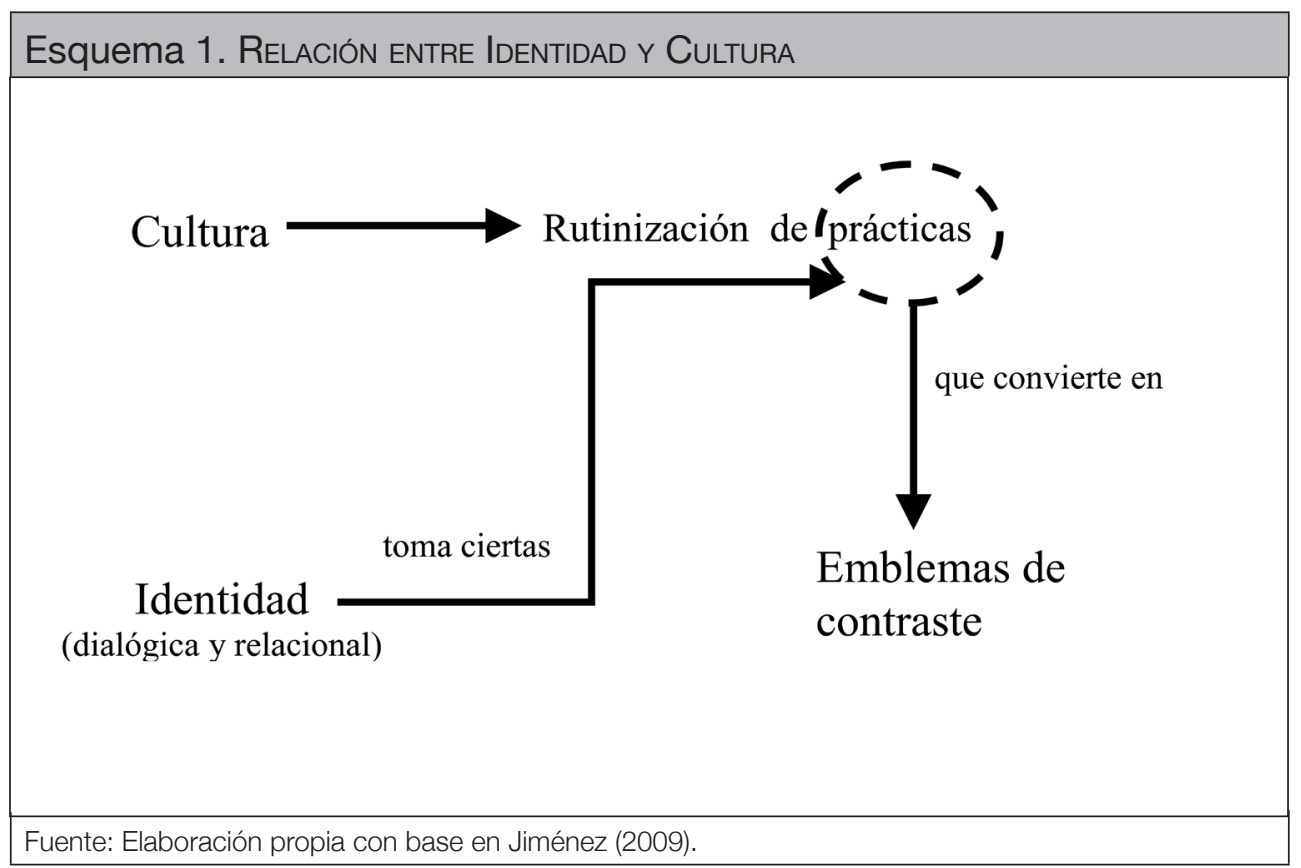

\section{La construcción del diálogo entre el saber tradicional y el conocimiento científico para reflexionar conjuntamente sobre la pertenencia étnica}

En el taller desarrollado el 13 de noviembre de 2013 en Suretka, Talamanca Bribri, se desarrolló un proceso de diálogo con una dinámica específica que detallamos a continuación. Al taller asistieron más de 30 maestros de Lengua y Cultura 4 . En un primer momento se expusieron por parte de los investigadores los conceptos de cultura e identidad, de forma tal que los docentes tuvieran mayor claridad en ciertos aspectos de dichos conceptos que hemos considerado fundamentales, sus diferencias y relaciones, lo cual se pudo realizar efectivamente a través de las síntesis esquemáticas montadas en una presentación visual (en formato Office Power Point).

Posteriormente reprodujimos una síntesis con los principales elementos que los docentes de Lengua y Cultura nos definieron en momentos anteriores (talleres previos, y entrevistas cualitativas específicas aplicadas con algunos de los docentes) como elementos centrales que forman parte de su identidad indígena. La información en bruto se configuraba como una lista de elementos

$4 \quad$ Agradecemos a la lingüista Carmen Rojas de la Unidad de Educación Indígena del MEP, quien nos permitió la programación del espacio del taller para el 13 de noviembre de 2013, aprovechando una convocatoria de varios días efectuada por esta misma instancia, de forma que no se interfirió con el calendario anual que rige el trabajo de estos maestros de Lengua y Cultura. 
y rasgos culturales diversos, que para efectos del presente artículo la sintetizamos en varias categorías que explicamos en el Cuadro 1.

Cabe resaltar que la mayoría de los rasgos enunciados son temas y contenidos que estos maestros desarrollan en sus clases. Desde la perspectiva de los docentes de Lengua y Cultura, el trabajar estos aprendizajes le permiten a las nuevas generaciones reforzar su identidad, porque así conocen sus raíces y desarrollan la valoración positiva de las mismas. Para ellos, generar espacios escolares para apreciar los conocimientos que sus padres y abuelos han transmitido de manera oral por generaciones, es algo que otorga mucha importancia a las lecciones de Lengua y Cultura.

En un tercer momento se sometieron a discusión los elementos por ellos previamente definidos y expuestos en el Cuadro 1. Así, por ejemplo, se les preguntó: ¿están seguros de que la lengua es un elemento central que determina la identidad de ustedes? Respondieron que sí. Seguidamente se les preguntó: ¿alguien conoce gente "no indígena" que habla bribri o cabécar? A lo cual respondieron positivamente también, porque de hecho existen casos de personas nacionales y extranjeros ("hay gringos que hablan muy bien nuestra lengua" decían algunos) que hablan alguna de esas lenguas ${ }^{5}$. ¿Entonces por hablar bribri o cabécar, esas personas pueden ser consideradas bribris o cabécares? En esta última pregunta respondieron que no. Y ahí es donde los docentes se pusieron a dudar si la lengua era un elemento que realmente determina la pertenencia étnica en el caso de sus culturas. Y, evidentemente, no era el caso. Igual se procedió con la artesanía, bajo el ejemplo hipotético que un no indígena perfectamente podría aprender las técnicas de manufactura de artesanías, pero esto no lo convertiría necesariamente en bribri o cabécar. La artesanía comenzaba a ser descartada de la lista también.

Al tomar en cuenta las nuevas consideraciones y la definición conceptual sobre cultura e identidad facilitada al inicio de la dinámica, se les propuso que trataran de determinar de nuevo y con base en la lista definida por ellos mismos, cuál o cuáles eran los rasgos que verdaderamente generaban la distinción de una identidad exclusivamente bribri o cabécar. Rápidamente, los maestros señalaron, de manera unánime, al sistema clánico como el rasgo fundamental: para ser bribri o cabécar hay que pertenecer a uno de los clanes existentes, que se hereda por la vía materna.

5 En la gran mayoría de los casos se trata de lingüistas o antropólogos, nacionales o extranjeros, que han hecho investigaciones sobre dichas culturas y han aprendido las lenguas indígenas. 


\section{Cuadro 1. Principales Rasgos Culturales enUnCIAdos POR LOS DOCENTES DE} LENGUA Y CULTURA QUE AFIRMAN LA IDENTIDAD ÉTNICA

\begin{tabular}{|l|l|}
\hline Rasgo cultural & Descripción \\
\hline $\begin{array}{l}\text { Mitología e historia } \\
\text { oral }\end{array}$ & $\begin{array}{l}\text { En esta categoría agrupamos todo lo que se relaciona con las historias sobre el } \\
\text { origen del mundo, de los seres humanos, las plantas, los animales y las cosas }\end{array}$ \\
\hline
\end{tabular}
que existen en el mundo.

Para los maestros de Lengua y Cultura, la mitología, es una temática que se comparte en los salones de clase y con la que las comunidades indígenas se identifican. Los mitos (no en su sentido vulgarizado como "algo que no es verdadero", sino en el sentido de la antropología y de la historia de las religiones, en tanto que "historia sagrada") son entendidos como una narración que describe y retrata en lenguaje simbólico, el origen de los elementos y supuestos básicos de una cultura. A partir de estas narraciones se originan y se da sentido a todas las costumbres, rituales, leyendas, historias que en la actualidad se conservan como una forma de recordar sus orígenes.

Se mencionó que en las culturas bribri y cabécar, el ambiente y la forma de organizarse socialmente son elementos identitarios, que están íntimamente ligados a su mitología. En la cosmovisión bribri y cabécar, el supramundo y el inframundo están complejamente entrelazados y, a la vez, cada uno compuesto por diversas etapas. Así, por ejemplo, todos los animales en el mundo real tienen un dueño que vive en el inframundo, quien ve de diferente modo a dichos animales (ejemplo: al tepezcuintle lo ve su dueño como un ayote; el zaíno se compara con un tiquizque, el pescado se relaciona con una yuca, etcétera).

Las menciones sobre el rancho tradicional las hemos incorporado aquí, en virtud de que la mitología dota de sentido dicho aposento, sus diferentes partes y su modo de construcción. Así, por ejemplo, en la construcción de una casa, los amarres con bejuco, son comparados con estrellas y, al igual que estos ejemplos, cada elemento en sus vidas está en relación con pasajes de la mitología cosmogónica.Fueron variadas las referencias al ser supremo omnipresente según el universo espiritual de estos pueblos indígenas; el dios Sibô. Se resaltan las historias sagradas que narran la antropogénesis: Sibô hizo a los primeros indígenas de semillas de maíz, trayendo las semillas de un lugar llamado Sulàkạ́ska, ("el Lugar del Destino"). Las semillas traídas por Sibö eran granos de maíz negro, amarillo, blanco y morado, lo que explica la diversidad de la especie humana.

Además del ser supremo, se hizo referencia a la gran cantidad de seres protectores que existen en la concepción espiritual de bribris y cabécares. Por ejemplo están los encargados de velar por los cultivos como "Duwàlök" que es el rey de los animales. "Yëria", que también puede significar cazador, es un ser que se encarga de cuidar a todos los animales que hay en el bosque y castiga a aquellas personas que abusan de ellos con su arco y flecha que se convierte en culebra (también se le llama Shulákama).

Referencias con respecto a los puntos cardinales. La salida del Sol por un punto del horizonte y su desaparición por el opuesto permitió al hombre disponer de estos puntos como referencia de ubicación. Los bribris y cabécares utilizan el este y el oeste como referencia y los vinculan con su mitología. Así, por ejemplo, los primeros seres malignos que tenían que ser expulsados o tenerlos lejos de los indígenas los enviaban al este, mientras que, cuando una persona muere se entierra con la cabeza hacia donde se oculta el sol, esto para que pase la prueba hasta el "Sulàkáska".

Se destaca igualmente la interrelación entre extracción de productos y los valores tradicionales. Sibô dejó los animales para el alimento, pero no hay que abusar, porque tiene que haber un equilibrio. En la mitología de esta cultura se tiene la idea de que cada animal tiene un dueño el cual es su protector, entonces se cree que si, por ejemplo, se va a pescar, hay que decir que se va a sacar yuca, por respeto al dueño de ese animal y para que así permita la cacería efectiva; si no se toma esta previsión, el dueño de los peces los esconde y no se consigue nada. 
Cargos tradicionales
Se mencionaron los cargos tradicionales, que están directamente relacionados con la mitología. Según la tradición, Sibö trajo las semillas para crear a los seres humanos durante la noche, motivo por el cual, el curandero, llamado awá en bribri, y jawá en cabécar, solo realiza sus cánticos y curaciones de noche. Además del Awá o Jawá, existen otros especialistas que conocen algunos cantos riturales, como Óköm (encargado de rituales fúnebres) y Bikákala (maestro de ceremonias), entre otros. Hay cantos para sanar enfermedades, para ceremonias rituales como funerales, en festividades como nacimientos, siembras, fenómenos naturales, huracanes, entre otros. Dependiendo de la actividad, estos cantos se acompañan de plantas, semillas, piedras, entre otros, que le permiten a los conocedores de este tema contribuir con la actividad que se desarrolla. Los especialistas tradicionales de estas culturas deben pertenecer a un clan específico que les posibilita realizar sus respectivas actividades.

Artensanía autóctona

La mayor parte de la artesanía está confeccionada a partir de material vegetal, generalmente fibras de plantas presentes en la zona, algunas de ellas teñidas con tintes naturales, se utilizan para hacer los diferentes tipos de canastos, desde los que se usan para trasladar semillas, yuca, ñame y otros alimentos hasta los que emplean las personas dedicadas a realizar las curaciones.

Se mencionó que el diseño de las jícaras hace referencia a la naturaleza. Estos utensilios están cargados de plantas y animales propios de la zona, lo que a su vez relacionan con la mitología; si el diseño es un gavilán, se recuerda la historia del animal que se comía a las personas, si el diseño es una culebra se hace referencia a la historia del Tkabëköl (la serpiente que castiga a las personas que cometían incesto), el tigre representa el clan de los Uséköl (sumo sacerdote, máxima autoridad), clan superior que cuida a los demás clanes.

Se dio incluso una lista donde se clasifican los productos artesanales según el significado de su diseño y función:

-Chácara: se elabora con dibujos de paisajes que representan la naturaleza.

-Jícaras: los animales considerados sagrados como los dantos, tigres y animales en vía de extinción de la comunidad.

-Canastos: las esquinas representan los cuatro puntos cardinales y la estructura significa el origen de los indígenas después de ser devorados por lablu (parásitos). -Batea: la utilidad es para plato, para almacenar y colar arroz, café y pejibaye.

-Tambor: significa la creación del sol. No se puede hacer a la luz pública ni tampoco por una mujer embarazada, se corta el material en cuarto creciente.

-Maraca: se usa en ceremonias de funerales por los cantores, los dibujos representan la naturaleza y los animales que existen.

-Pilón: se confecciona de un árbol como cedro macho y se corta en cuarto menguante.

-Bika': se confecciona con fibras de bijagua y esto se utiliza por los awá en las curaciones y ritos, se corta en octubre.

-Hamaca: se confecciona con cabuya y su significado es el nacimiento de los reyes de las ardillas.

-Cerbatana: se fabrica con una clase de material que se encuentra en zonas de clima frío. Se confecciona guardando mucha dieta y no acercarse al fogón mientras se esta haciendo.

-Arco: significa el arma con que cazaba Sibö en la época de la creación del mundo. -Flecha: su significado representa la serpiente y se confecciona con fibras de pejibaye. -Güirro: se confecciona con bambú y lo utilizan para ceremonias en funerales.

-Banca: se confecciona con madera de cedro y laurel. Su utilidad es para hacer ritos y cantos de los awá. La figura de tortuga representa la llegada de Yëria a la casa del rey de los animales. 


\begin{tabular}{|c|c|}
\hline Sistema clánico & $\begin{array}{l}\text { Además de ser referido, este elemento fue relacionado por los docentes con los } \\
\text { dos anteriores. La organización social por medio de clanes, constituye una parte } \\
\text { muy importante de su identidad. } \\
\text { La organización clánica introduce una serie de elementos de las sociedades bri- } \\
\text { bris y cabécares: hay una relación entre el clan y los cargos tradicionales que un } \\
\text { individuo puede desarrollar. Igualmente, cada clan implica una serie de facultades } \\
\text { así como de limitaciones; por ejemplo, hay clanes que tienen prohibido comer } \\
\text { carne de danta. } \\
\text { Para los maestros de Lengua y Cultura es de suma importancia que el niño o } \\
\text { la niña conozca el clan al que pertenence para que desarrolle su sentido de } \\
\text { identidad indígena. Los niños deben conocer desde muy pequeños a qué clan } \\
\text { pertenecen, ya que esto además de identificarlos como parte de un grupo, evita } \\
\text { entre otras cosas, que se cometa incesto y le permite saber con cuáles clanes } \\
\text { puede establecer familia a futuro. } \\
\text { Cada clan tiene una función que solo ese clan puede realizar dentro de la socie- } \\
\text { dad, por lo que es de suma importancia que el niño sepa cuál es la labor que } \\
\text { puede llegar a desempeñar para su cultura, si acepta asumirla, además de que lo } \\
\text { ayuda a identificarse con un grupo familiar. }\end{array}$ \\
\hline La lengua indígena & $\begin{array}{l}\text { Se hizo referencia a los idiomas vernáculos bribri y cabécar como elementos de } \\
\text { su identidad. }\end{array}$ \\
\hline $\begin{array}{l}\text { Los valores tradicio- } \\
\text { nales }\end{array}$ & $\begin{array}{l}\text { Se mencionaron los valores inculcados por los antecesores principalmente por los } \\
\text { ancianos y ancianas como uno de los pilares de estas culturas. Se fundamentan } \\
\text { en la unidad, solidaridad y respeto para con la comunidad. Están relacionados } \\
\text { con la mitología. Los encargados de mantener la tradición oral sagrada son los } \\
\text { awá y jawá, es decir, los chamanes considerados los especialistas de la medicina } \\
\text { autóctona y de la literatura oral, que se comparte en momentos llenos de signifi- } \\
\text { cado con la finalidad de que esta no muera y que los valores perduren. } \\
\text { La vivencia de valores está presente en todas las actividades como la cacería, la } \\
\text { cocina, la construcción de chozas, entre otras. Es importante destacar cómo uno } \\
\text { de los valores más importantes que se rescatan en estas culturas es el amor por } \\
\text { la naturaleza, lo cual se denota en la forma en que sus mitos, leyendas y vivencias } \\
\text { compartidas resaltan, generalmente, su respeto por lo natural y la necesidad de que } \\
\text { se mantenga el equilibrio entre el ambiente, el mundo espiritual y el ser humano. }\end{array}$ \\
\hline
\end{tabular}

Los investigadores del proyecto anticipábamos esa conclusión. En términos generales, de manera explícita bribris y cabécares constantemente refieren que el clan es un elemento fundamental para determinar si una persona es realmente bribri o cabécar. Las numerosas entrevistas e interacciones a lo largo del tiempo con personas indígenas de estos territorios, nos lo evidenciaban. Pero ¿era solamente el clan?

Acto seguido se les preguntó ¿qué es lo que hace que el clan sea heredado por la vía materna en el caso de los bribris y cabécares? Al principio no entendieron la pregunta; daban por sentado que el sistema clánico preestablecía que fuera por la vía materna. Pero les rebatimos esa creencia cuando se les dio ejemplos de culturas en otras partes del mundo que también se regían por sistema de clanes, pero que a diferencia de ellos, eran heredados por la vía paterna; tal es el caso de los pueblos bantú en África (diseminados en las regiones de Camerún, África Central, África del este y África del sur), los muong de Vietnam (en el continente asiático) o los tharu de la India, entre otros. Ante 
la nueva evidencia, los maestros guardaron silencio, mostrando un semblante de intriga ante la nueva interrogante: ¿qué es lo que hace que el sistema clánico en unas culturas sea heredado por la vía paterna, mientras que en otras culturas es heredado por la vía materna? No hallaban la respuesta.

A continuación se les expuso un rasgo que no habían enunciado, porque se trata de una institución que, si bien es cierto existe en todas las culturas del mundo, no siempre es considerada de manera explícita por los miembros de una cultura, aunque forme parte de la estructura que fundamenta la organización social: nos referimos al sistema de parentesco. El sistema de parentesco es una institución que la antropología ha estudiado desde sus inicios como disciplina científica. Esta institución, en términos generales, es la que define las relaciones entre los parientes en una cultura dada; establece relaciones parentales por consanguinidad (por ejemplo, entre padre-hijo, hermano-hermana) o por afinidad (por ejemplo, entre cónyuges, entre suegro y yerno, entre cuñados). Además de las relaciones entre parientes, el sistema de parentesco define también la ascendencia-descendencia y las relaciones de afinidad permiten establecer las potenciales parejas. En el caso de bribris y cabécares, el sistema de parentesco es de base iroquesa (Bozzoli, 1979), según la tipología tradicional establecida en antropología. Este tipo de parentesco implica relaciones de afinidad específicas que difieren de otros sistemas y se caracteriza porque, entre otras cosas, permite el matrimonio entre primos cruzados (porque no son considerados como familia), mientras que los primos paralelos son considerados como hermanos, por lo que es prohibido formar pareja con ellos. En efecto, este sistema de parentesco juega un papel central en la determinación de la herencia matrilineal de los clanes.

La combinación entre el sistema de parentesco, que fundamenta la matrilinealidad y el sistema clánico para la determinación de una identidad bribri o cabécar, ya había sido constatada por la antropología costarricense. En 1986, el antropólogo Marcos Guevara Berger comprueba, efectivamente, que la pertenencia étnica de bribris y cabécares se define por adquisición de una identidad clánica, lo cual, a su vez, presupone una madre que la tiene por efecto directo de la estructura de parentesco; asimismo, este antropólogo en su investigación constata que la lógica del orden familiar en realidad no está determinada unívocamente por los clanes, sino que, en realidad, la estructura clánica es consecuencia del orden que establece el parentesco, aunque ciertamente se implican entre sí (Guevara Berger, 1986). Por supuesto que, como bien lo ha explicado este antropólogo ${ }^{6}$, tal constatación la pudo hacer en parte gracias al trabajo previo que ha realizado María Eugenia Bozzoli, entre otros referentes, con su esbozo del sistema de parentesco de los bribris y su fundamento matrilineal, pues el hecho de que ella haya sistematizado el sistema de

$6 \quad$ En comunicación personal con Federico Guevara Víquez, año 2013. 
parentesco implicó que él no tuvo que hacerlo, por lo que pudo dedicarse al análisis de cómo encajaba la lógica clánica dentro del mismo.

Luego de la exposición de estos datos, los maestros de Lengua y Cultura estuvieron de acuerdo con la constatación hecha por el citado antropólogo y agradecieron la información. Vieron con mucha coherencia el hecho de que el sistema de parentesco (que tradujeron en bribri como "yàmi pa": literalmente "parientes") se complementara con el sistema clánico (que tradujeron en bribri como "ditséwö") para la determinación de la pertenencia étnica. No tuvieron inconvenientes para comprender la explicación sobre los sistemas de parentesco porque siempre han tenido muy claro que el sistema familiar de ellos es distinto al de los costarricenses no indígenas.

Finalmente, se hizo un cierre en conjunto en el que se avaló una representación esquemática que sintetiza la reflexión sobre las bases de la pertenencia étnica entre bribris y cabécares expuesta en el Esquema 2.

Esquema 2. INSTITUCIONES CULTURALES QUE DETERMINAN LA PERTENENCIA ÉTNICA BRIBRI Y CABÉCAR

Sistema clánico
"ditséwö"
Sistema de parentesco
"yàmípa"

Cerramos este apartado refiriendo las palabras de un señor mayor y gran intelectual bribri, don Alejandro Swaby Rodríguez, con quien efectuamos una entrevista específica en el marco del proyecto:

Nuestra historia dice que nosotros nacimos o aparecimos en un principio en una montaña que está cerca por aquí, que se llama Sulayöm; "Sula'” en la lengua 
indígena es un Dios7, "yöm” significa que Dios nos formó ahí, ¿no?, entonces eso es la base. Por eso es que nosotros creemos de dónde venimos, sabemos quiénes somos precisamente por los clanes, nuestros clanes. Eso es como un medio histórico que nos lleva hasta el final de nuestras vidas. Por ejemplo, decimos que las mujeres son ditsökatá. Ditsökatá significa que son base, base principal de lo que son nuestros clanes ¿no? Las mujeres son las que siguen eternamente los clanes, los hombres por ejemplo no. Los hombres que se casan con otra pareja, pues los clanes de él, hijos de él tienen que ser de la pareja, por ejemplo yo soy kolkuâk [clan del dueño del guarumo], hay tu'bulwak [dueño del ñame] si mi hijo se me casa con una tu'bulwak entonces los hijos de él serán tu'bulwak, y si un tu'bulwak se casa con una kolkuâk sus hijos serán kolkuâk, ¿me entienden? Entonces esas son las leyes divinas que nos rigen a nosotros (entrevista con Alejandro Swaby Rodríguez, 7 de marzo de 2013).

En efecto, el sistema clánico es un elemento central, explícita y constantemente enunciado por miembros de estas culturas, en la determinación de la identidad indígena. Sin embargo, y tal como se percibe, de forma implícita, en la explicación de Alejandro Swaby, no se trata solo del sistema clánico. Aparece también en este particular proceso de adhesión/exclusión el sistema de parentesco, que establece la descendencia en el caso de bribris y cabécares, de forma matrilineal.

\section{Últimas reflexiones}

Después de valorar, por un lado, la voz propia de bribris y cabécares sobre cómo se establece la adscripción étnica en el caso de estos pueblos y, por otro lado, el abordaje analítico de la identidad bribri y cabécar desde la antropología, reconocemos comprensivamente la complementariedad entre dos instituciones específicas -el sistema clánico y el sistema de parentesco- que se imbrican en la determinación de la adscripción cultural de bribris y cabécares. Son estas dos instituciones las que se complementan para asentar las bases de la identidad étnica de estos dos pueblos indígenas. A su vez, los maestros de Lengua y Cultura avalaron y validaron apreciaciones de la disciplina académica, una vez que se les empodera con los referentes necesarios. Comprendieron más cabalmente las diferencias entre cultura e identidad y, al mismo tiempo, constataron que hay elementos de estos fenómenos que son más implícitos e inconscientes; no todos los rasgos son instrumentalizables ni se muestran tan evidentes para la deliberación individual o colectiva, cuando se habla de identidad y de cultura.

Es posible generar diálogo entre el saber tradicional y el conocimiento científico. La experiencia descrita en este artículo así lo confirma. Los investigadores de

7 En realidad Sula' no es estrictamente un dios, sino, un artesano, ayudante de Sibö. Esto de seguro lo tiene bien claro don Alejandro Sweaby. No obstante, lo expuso en esos términos muy probablemente para simplificarnos la explicación, suponiendo que no teníamos mucho conocimiento sobre las historias sagradas talamanqueñas. 
las universidades debemos hacer esfuerzos por generar ese diálogo y asumirlo como un compromiso permanente con las poblaciones con las cuales trabajamos. La visión clásica academisista que distancia el conocimiento científico del conocimiento vernáculo o tradicional debe ser superada y no debe seguir siendo reproducida por posturas que valoran el primero como superior al segundo, ni por posturas que niegan los aportes que, efectivamente, se pueden extraer de la inmensa cantidad de investigaciones publicadas en tesis o artículos científicos, desde diversas disciplinas y que se hallan en su mayoría dentro de los centros de documentación de las casas de educación superior. Es cierto que muchos de los productos y hallazgos científicos se encuentran en formatos o lenguajes técnicos establecidos por las diferentes tradiciones académicas, que dificultan el acceso y la comprensión para personas que se hallan fuera de la vida universitaria. Pero entonces por parte de los investigadores y académicos se debe hacer el esfuerzo por tender los puentes necesarios para que se dé el acercamiento con esos conocimientos y hallazgos, para que se puedan valorar según su pertinencia e implicaciones para las comunidades que muchas veces se sienten vulneradas por ser "objetos de estudio", pero que no siempre las hemos hecho sentirse parte activa del proceso de construcción del conocimiento. Asimismo, cuando hacemos este esfuerzo, es algo que las comunidades con las que trabajamos agradecen enormemente.

\section{Referencias}

Bartolomé, Miguel A. (2006). Los laberintos de la identidad. Procesos identitarios en las poblaciones indígenas. Avá, (9), 28-48.

Bozzoli de Wille, María Eugenia. (1979). El nacimiento y la muerte entre los bribris. San José: Editorial Universidad de Costa Rica.

Guevara Berger, Marcos. (1986). Mythologie des Indiens Talamanca (Costa Rica) (Tesis de doctorado en Etnología y Sociología Comparada). Université de Paris X-Nanterre, París, Francia.

Guevara Víquez, Federico y Ovares Barquero, Sandra. (2013). Reconociendo modelos autóctonos de enseñanza y aprendizaje en los pueblos bribri y cabécar. En I/ Coloquio Latinoamericano Colonialidad/Decolonialidad del Poder/Saber/Ser Educación, Pedagogía y Cultura, 7-8 de octubre 2013 (memoria sin publicar). Heredia, Costa Rica: Universidad Nacional de Costa Rica.

Instituto Nacional de Estadística y Censo, INEC. (2014). Sistema de Consultas. Recuperado de http://www.inec.go.cr/Web/Home/GeneradorPagina.aspx

Jiménez Naranjo, Yolanda. (2009). Indeterminación conceptual de las prácticas educativas interculturales: los conceptos de cultura e identidad a examen. 
Estados plurales: Ios retos de la diversidad y la diferencia. México: Universidad Autónoma Metropolitana.

López, Luis E. (2001). La cuestión de la interculturalidad y la educación latinoamericana. En Seminario sobre prospectivas de la Educación en la Región de América Latina y el Caribe, 23-25 agosto 2000 (sin memoria). Santiago, Chile: Oficina Regional de Educación de la UNESCO. Recuperado de http://adei.org.pe/anexos/Interculturalidad/cuestion_latinoamericana.pdf

UNICEF. (2006). Niñez y Adolescencia indígena en Costa Rica: su derecho a la salud y a la educación. San José: UNICEF. 